\title{
Mesa de corte por plasma automatizada mediante LabVIEW
}

\author{
Automated plasma cutting table using LabVIEW \\ CASTILLO-QUIROZ, Gregorio †*, VARGAS-CRUZ, Juan Javier, REYES-LEON, Ivan y GONZAGA- \\ LICONA, Elisa
}

Ingeniería Mecatrónica, Instituto Tecnológico Superior de Huauchinango-ITSH-TecNM, Av. Tecnológico No 80, 5 de Octubre, Huauchinango, Puebla, México. C.P. 73173

ID $1^{\text {er }}$ Autor: Gregorio, Castillo-Quiroz / ORC ID: 0000-0002-1904-4172, CVU CONACYT ID: 162009

ID $1^{\text {er }}$ Coautor: Juan Javier, Vargas-Cruz / ORC ID: 0000-0003-3300-8837, CVU CONACYT ID: 904038

ID $2^{\text {do }}$ Coautor: Ivan, Reyes-Leon / ORC ID: 0000-0003-1294-0772, CVU CONACYT ID: 903594

ID $3^{\text {er }}$ Coautor: Elisa, Gonzaga-Licona / ORC ID: 0000-0001-5745-6330, CVU CONACYT ID: 904035

\begin{abstract}
Resumen
La técnica del corte por plasma mejor conocida por su simplicidad y capacidad para cortar prácticamente cualquier metal. Esta técnica tiene presencia en la mayoría de las industrias, con el paso del tiempo ha sufrido mejoras tecnológicas que permiten realizar cortes con una mejor calidad y una mayor productividad, por ello la importancia de realizar un sistema automatizado para realizar de manera eficiente y segura ésta tarea. El presente artículo muestra cómo se realizó la automatización de una mesa de corte por plasma utilizando la plataforma de desarrollo Motion y el lenguaje de programación gráfico LabVIEW de National Instruments. La interfaz que fue desarrollada brinda las herramientas necesarias para que el usuario pueda realizar de forma automática los cortes de diferentes materiales para la fabricación de piezas mecánicas. Con esta mejora se disminuye el riesgo de accidentes que implica el propio proceso, garantizando un buen funcionamiento y medidas de seguridad durante su operación. Adicionalmente se integraron señalamientos visuales que permiten conocer el estado de operación y funcionamiento de la mesa, así como botones de paro de emergencia.
\end{abstract}

Automatización, Cortes, Labview

\begin{abstract}
The plasma cutting technique best known for its simplicity and ability to cut virtually any metal. This technique is present in most industries, with the passage of time has undergone technological improvements that allow cutting with better quality and greater productivity, so the importance of an automated system to efficiently and safely perform this homework. This article shows how the automation of a plasma cutting table was carried out using the Motion development platform and the LabVIEW graphic programming language from National Instruments. The interface that was developed provides the necessary tools so that the user can automatically make cuts of different materials for the manufacture of mechanical parts. With this improvement, the risk of accidents involved in the process itself is reduced, guaranteeing good operation and safety measures during its operation. Additionally, visual signs were integrated that allow to know the operation and operation status of the table, as well as emergency stop buttons.
\end{abstract}

Automation, Cuts, Labview

Citación: CASTILLO-QUIROZ, Gregorio, VARGAS-CRUZ, Juan Javier, REYES-LEON, Ivan y GONZAGA-LICONA, Elisa. Mesa de corte por plasma automatizada mediante LabVIEW. Revista de Aplicaciones de la Ingeniería. 2019. 6-19: 110

\footnotetext{
* Correspondencia del Autor (Correo electrónico: gcquiroz1977@gmail.com)

$\dagger$ Investigador contribuyendo como primer autor.
} 


\section{Introducción}

El corte industrial de materiales como aceros no aleados, de aleaciones ligeras ha evolucionado según la demanda industrial. En el mercado existen diferentes herramientas para realizar el corte de diferentes materiales, como son las sierras mecánicas, máquinas de corte por láser, corte por electroerosión, método de corte con chorro de agua abrasivo, entre otros métodos. Dependiendo de las necesidades del usuario se selecciona la técnica de corte más adecuada, pero las necesidades de cortar diversos materiales de aleaciones diversos, diferentes calibres y velocidad en los cortes, ha obligado buscar alternativas más eficaces, una de ellas ha sido el corte por plasma. La técnica del corte por plasma es mejor conocida por su simplicidad y capacidad para cortar prácticamente cualquier metal, se ha convertido en un recurso aceptado para varias aplicaciones. Ésta técnica ha sufrido constantes desarrollos y mejoras a lo largo de la historia:

La tecnología de uniones de piezas metálicas por arco eléctrico vio sus éxitos en 1930 al construir un barco totalmente soldado en Carolina del Sur en Estados Unidos, años después se introdujo mejoras en el proceso como corriente alterna, y se utilizó protección como fundente granulado. En los años 40, se introdujo el primer proceso con protección gaseosa empleando un electrodo no consumible de wolframio y helio como gas protector, recibió el nombre de TIG (Tungsten Inert Gas).

El corte con plasma ha recorrido un largo camino desde que fue desarrollado a finales de los años 50 por ingenieros de Unión Carábido Corp. Actualmente es uno de los procesos de corte de placa metálica ampliamente utilizado para una gran variedad de industrias.

En 1954 los científicos descubren que, al aumentar el flujo del gas y reducir la abertura de la boquilla utilizada en la soldadura TIG, se obtiene un chorro de plasma. Este chorro es capaz de cortar metales, lo que dio lugar al proceso de corte por plasma conocido hoy en día. Actualmente los avances de la ciencia, la técnica y los métodos de análisis estadístico, han dado un impulso extraordinario a la electromiografía. En 1957, el proceso de corte con plasma fue desarrollado y patentado por Union Carbide como una extensión del proceso de soldadura por arco de tungsteno con gas (GTAW).
Entre 1962-1967, se concluyeron varios desarrollos en diseño de consumibles y se diseñó la antorcha de flujo dual para ayudar a mejorar la vida de los consumibles y la calidad de corte en materiales no ferrosos. Posteriormente en 1968, se comercializó el proceso de inyección de agua. Este proceso permitió cortar bordes limpios de corte cuadrado y velocidades más rápidas, así como cortar aceros al carbón con una calidad de corte aceptable.

Entre los años 1970-1979, debutaron la mesa de agua y el amortiguador de agua, diseñados para brindar control de vapores y humos. Surgieron controles automatizados de altura basados en voltaje de arco para una calidad de corte más consistente y una vida más larga de las partes consumibles.

Años más tarde, se introdujeron sistemas de corte con plasma basado en oxígeno, que ayudaban a mejorar lo cuadrado de los bordes y la metalurgia de los bordes (un borde soldable más terso) y permitían cortar aceros al carbón a menores niveles de potencia y mayores velocidades de corte. En la década de los 80s muchos desarrollos en el proceso de corte con plasma de aire permitieron una mejor portabilidad y menores niveles de potencia para corte manual y corte mecanizado de hoja delgada.

En los inicios de los 90s, se desarrollaron diseños de fuentes de potencia que usaban salidas controladas por corriente y moduladas por ancho de pulso. Algunos sistemas empezaron a usar fuentes de potencia con tecnología de inversores más pequeños de peso más ligero, adecuadas para sistemas de plasma portátiles manuales.

En 1992 se introdujo la tecnología de proceso con oxígeno de larga vida. Se trataba esencialmente de un método controlado por microprocesador para controlar las presiones ascendentes del gas de plasma así como el amperaje de salida de la fuente de potencia. Ayudaba a aumentar la vida de las partes consumibles del corte con plasma de oxígeno entre cuatro y seis veces; mejoraba la consistencia de las partes; y ayudaba a reducir el costo del corte con plasma. 
En 1993 se desarrolló el plasma de alta definición, una técnica que requirió la implementación de la tecnología del oxígeno de larga duración. Este proceso permitió un nuevo diseño de boquilla que aumentó la densidad de energía de un arco de plasma de oxígeno hasta cuatro veces, permitiendo cortes más cuadrados y más limpios en todos los espesores de material.

Tres años más tarde surgieron los sistemas automatizados de control de flujo de gas. Éstos se conectaban digitalmente con los $\mathrm{CNC}$ de las máquinas. Estos controles de flujo de gas eliminaron en parte la posibilidad de errores relacionados con el operador de la máquina en el ajuste de parámetros para el proceso de corte.

Entre 1996-2006 ocurrieron muchos desarrollos relacionados con la mejora de la calidad de corte y la productividad, y con la automatización de muchos parámetros de corte del proceso. Éstos incluyeron el plasma integrado, un sistema que acoplaba estrechamente el CNC, la fuente de potencia del plasma, el control de flujo de gas, el software CAM y el sistema de control de altura para automatizar el proceso. Con esta característica integrada al sistema, el trabajo del operador de la máquina se volvió mucho más simple, y el proceso dependió menos de la pericia del operador.

Actualmente se desarrollan nuevas tecnologías para automatizar completamente y coordinar las funciones que afectan la calidad de corte. La necesidad de realizar cortes de piezas con formas regulares e irregulares en el área de Tecnologías de Fabricación se hace cada vez más común, por lo que es necesario desarrollar un sistema de corte por plasma automatizado, que sea capaz de realizar diferentes formas de cortes de una manera eficiente, de mayor calidad y reducción de tiempo de fabricación. Además que proporcione una mayor seguridad al operador.
El presente trabajo contribuye a la automatización e instrumentación de una mesa de corte por plasma capaz de realizar diferentes formas de cortes en placas de acero de manera eficiente y mayor calidad, mediante el uso de LabVIEW de National Instruments con un desarrollo propio de programación modificable, instrumentación para el desarrollo de la mesa de corte, desarrollo de algoritmos para leer y decodificar la secuencia de movimiento de archivos CAD desarrollados en AutoCAD y SolidWorks y demás un diseño desarrollado de un interfaz final para el usuario.

La interfaz implementada brinda las herramientas necesarias para que el usuario pueda realizar de forma automática la fabricación de piezas mecánicas. Con esta mejora se disminuye el riesgo de accidentes que implica el propio proceso, garantizando un buen funcionamiento y medidas de seguridad durante su operación.

El desarrollo de este trabajo de investigación está dividido en las secciones que a continuación se describen:

1. Materiales: $\mathrm{Se}$ describen las características de los componentes utilizados en la construcción del prototipo.

2. Metodología: Se describen los pasos que se siguieron para el desarrollo de la investigación.

3. Resultados: En esta sección se analizan los resultados obtenidos para determinar si se ha logrado el objetivo.

4. Conclusiones: Se habla de los objetivos logrados satisfactoriamente y de cómo contribuye este trabajo en diversas aplicaciones relacionadas con el corte por plasma.

5. Agradecimientos: Se agradece a las personas e instituciones que permitieron el desarrollo de esta investigación.

\section{Materiales}

Para la elaboración de este proyecto se precisó del NI MID-7652 para el control de 2 ejes, sensores inductivos, encoders, motores DC como componentes utilizados para el sistema se explica en el esquema de la Figura 1. 


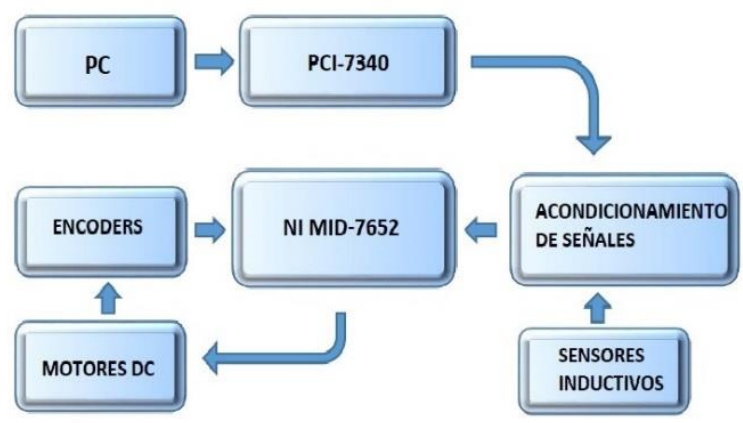

Figura 1 Esquema de componentes para el sistema Fuente: Elaboración Propia

\section{A. NI MID-7652}

El controlador brinda todas las características de un módulo cableado de interfaz de movimiento universal (UMI) con las mejoras de un drive de motor en un solo producto. Un solo cable blindado conecta los drives de potencia MID al controlador de movimiento, brindando la ruta para todos los comandos de motor, así como señales de E/S de movimiento, control y candidato a ser simulado a través del ambiente gráfico. En la Figura 2 se muestra el componente utilizado.

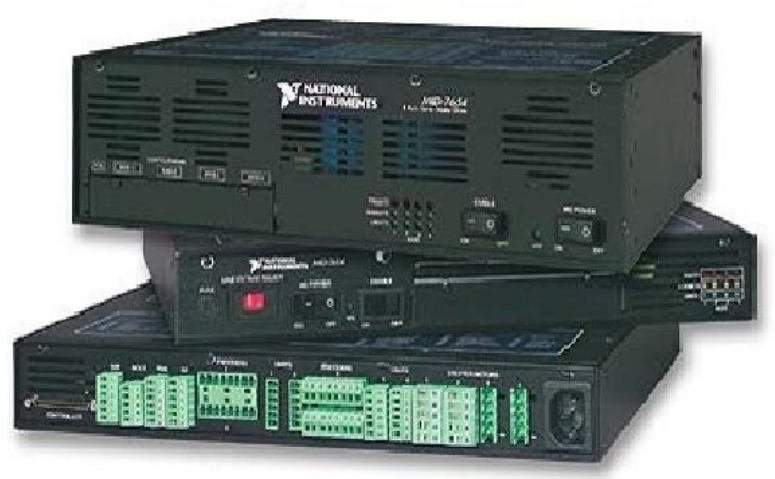

Figura 2 NI-MID-7652.

Fuente:http://sine.ni.com/nips/cds/view/p/lang/es/nid/385 4

\section{B. PCI-7340}

El controlador de movimiento NI 7340 es una combinación de servomotor y controlador de motor paso a paso para PXI, computadoras compactas de bus PCI y PCI. El controlador de movimiento NI 7340 proporciona totalmente control de movimiento programable para hasta cuatro ejes de movimiento independientes o coordinados, con E / S de movimiento dedicado para interruptores de límite e inicio y $E / S$ adicional para fines generales.
Este tipo de controladores ofrece características avanzadas tales como el control de trayectoria de movimiento combinado ya sea circular, lineal, de punto a punto, engranaje, y el control de vectores en el espacio, esto puede ser en la operación de movimiento integrado o entornos de programación host-centric, véase Figura 3.

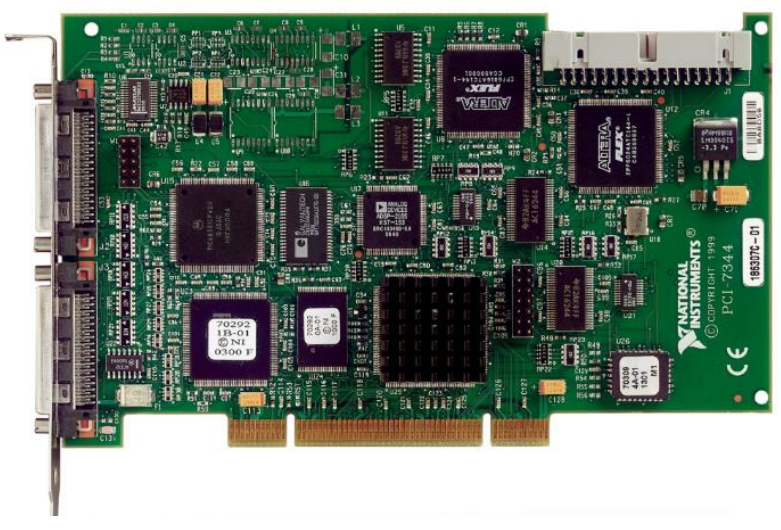

Figura 3 PCI-7340

Fuente:

https://www.ni.com/es-mx/support/model.pci-7344.html

\section{Sensor Inductivo}

Los sensores inductivos utilizados fueron OMRON E2EQ-X7D1-M1GJ diseñados para detectar la presencia de materiales metálicos. Estos detectan su presencia generando un campo electromagnético y detectando los cambios en este campo que son causados por la aproximación del objeto metálico. Los interruptores de proximidad inductivos consisten de una bobina de alambre, un oscilador, un rectificador (circuito detector) y un transistor (circuito de salida), véase Figura 4.

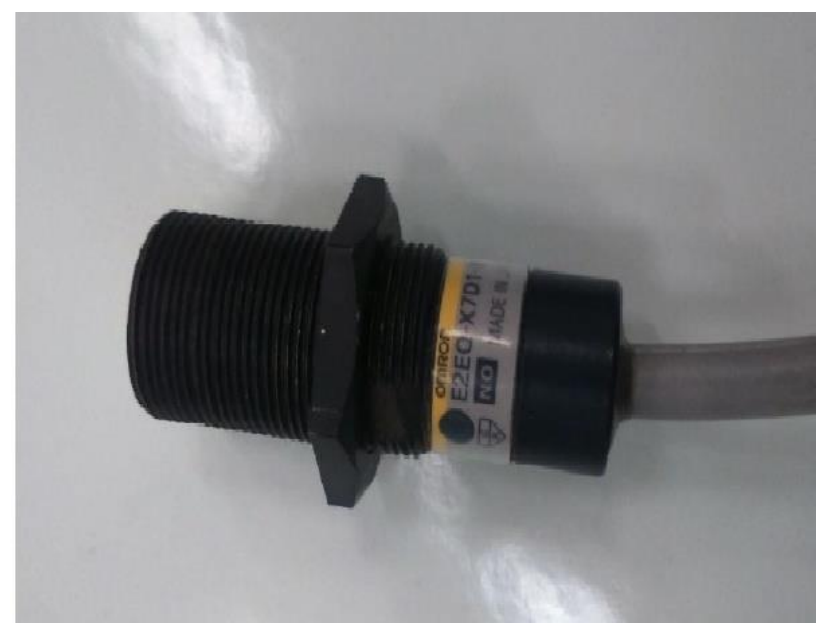

Figura 4 OMRON E2EQ-X7D1-M1GJ.

Fuente: Elaboración Propia 


\section{Encoder}

Para este proyecto se utilizó el encoder rotativo AMT102-V CUI INC que se muestra en la Figura 5, este encoder se monta sobre una pieza de aluminio que se ensambla en la parte trasera del motor, el encoder hace contacto con el eje que sobresale del motor esto para realizar la medición del desplazamiento.

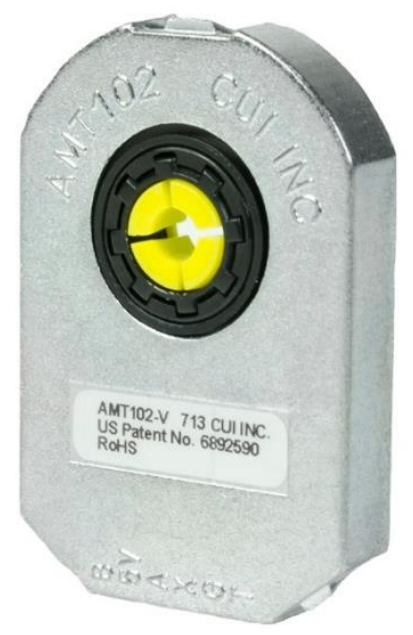

Figura 5 AMT102-V CUI INC.

Fuente: https://www.moonsindustries.com/p/encoderamt10-series/amt102-v-000004716140201160

\section{Metodología}

La metodología para la automatización y desarrollo del software de control se basó en el funcionamiento de los sistemas de corte de plasma existentes en el mercado, las cuales funcionan gracias a que son capaces de descifrar los algoritmos que generan programas de diseño de piezas mecánicas. La información que generan los softwares de diseño contienen todo lo necesario para generar trayectorias del corte, las cuales mediante programación se traduce a señales con la ayuda de drivers y sistemas traductores que le permiten a los servomotores posicionarse dentro de un plano 2D y seguir cada posición indicada.

Los sistemas automatizados existentes en el mercado no permiten la modificación del código fuente. La interfaz que se implementó en este trabajo permite controlar todo el sistema, el cual fue desarrollado en base a experimentaciones para poder entender el funcionamiento del sistema de corte, el cual permitió el desarrollo de este tipo de tecnología a un bajo costo. El desarrollo de esta investigación se llevó a cabo con base en el diagrama propuesto en la Figura 6, donde se muestra el proceso que debe seguirse.

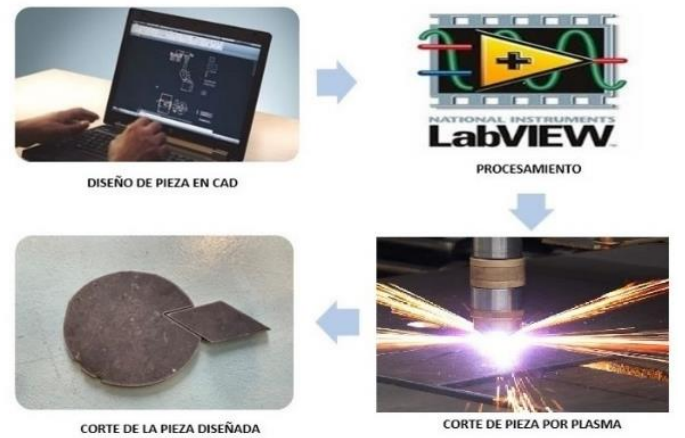

Figura 6 Proceso para cortes por plasma. Fuente: Elaboración Propia

Los pasos que se efectuaron son los siguientes:

\section{Acondicionamiento de señales}

Se realizaron las conexiones de todos los sensores hacia la placa acondicionadora de señales, la cual fue diseñada para moderar los voltajes hacia cada componente de acuerdo a los valores soportados, se trabajó con voltajes de 5 v y 24 v, véase Figura 7.

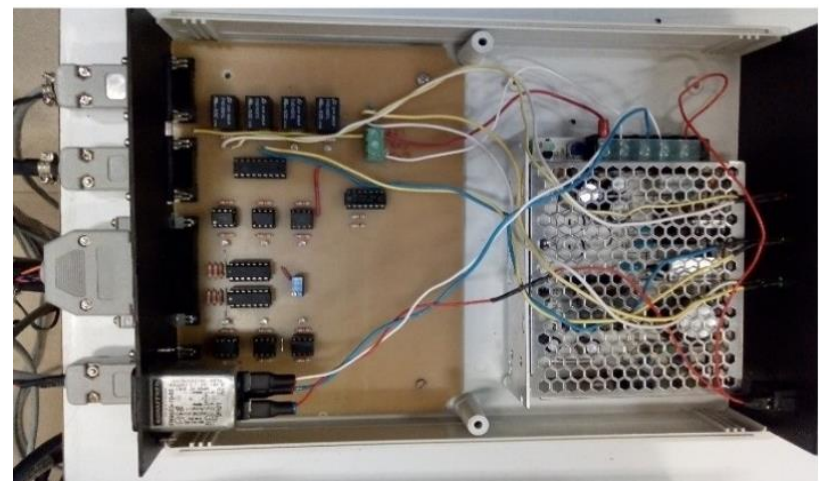

Figura 7 Placa para acondicionar señales. Fuente: Elaboración Propia

También se realizaron las conexiones hacia el MID-7652, como se muestra en la Figura 8 donde se puede apreciar que se colocaron etiquetas para identificar la procedencia de cada cable.

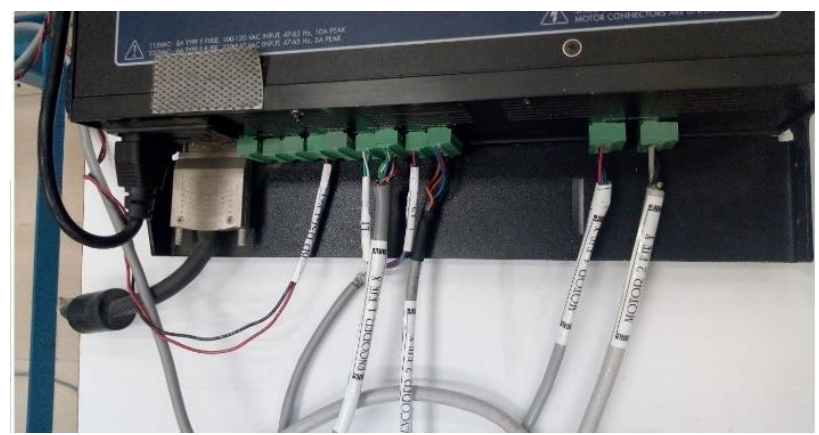

Figura 8 Conexiones hacia el MID-7652.

Fuente: Elaboración Propia

CASTILLO-QUIROZ, Gregorio, VARGAS-CRUZ, Juan Javier, REYES-LEON, Ivan y GONZAGA-LICONA, Elisa. Mesa de corte por plasma automatizada mediante LabVIEW. Revista de Aplicaciones de la Ingeniería. 2019. 


\section{Configuración de la corriente del MID-7652}

El MID-7652 cuenta con una serie de interruptores en los cuales se regula la corriente que se suministra a cada uno de los motores, desde la corriente entregada continuamente hasta la corriente pico, configuración para inhibir los motores en flanco de ascenso o descenso y configurar la inductancia como se muestra en la Figura 9.

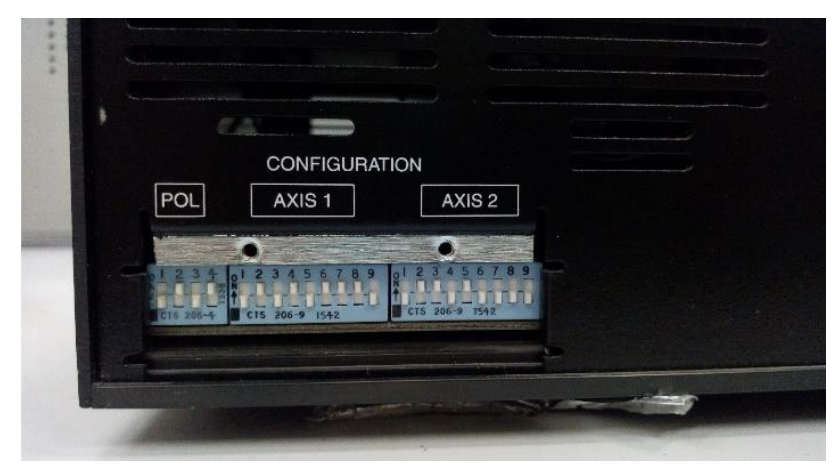

Figura 9 Configuración de corriente del MID-7652.

Fuente: Elaboración Propia

\section{Configuración de motores y la detección de sensores}

La configuración de motores se realizó mediante el software NI MAX, donde se configuró todos los parámetros necesarios de la tarjeta PCI 7340, véase Figura 10.

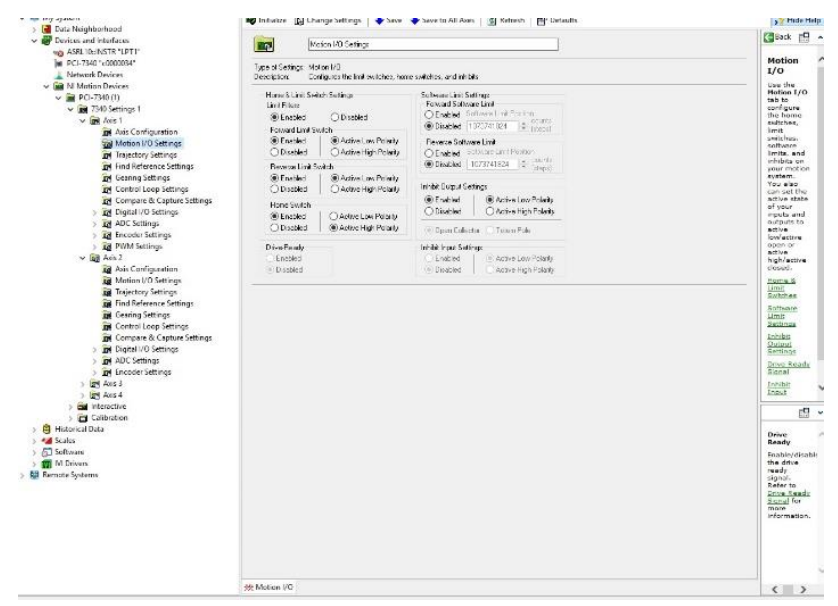

Figura 10 Proyecto en AVR Studio 4

Fuente: Elaboración Propia

\section{La programación en LabVIEW}

La programación para realizar los cortes se realizó mediante la plataforma LabVIEW, diseñándose mediante estructura de máquinas de estado en cola (véase Figura 11), que envía comandos y otros datos desde múltiples puntos de origen (puntos de producción), como los eventos de usuario y de uno o más procesos paralelos, y se maneja en un proceso de máquina de estado (punto de destino del consumidor) en el orden en que se agregaron a la cola.

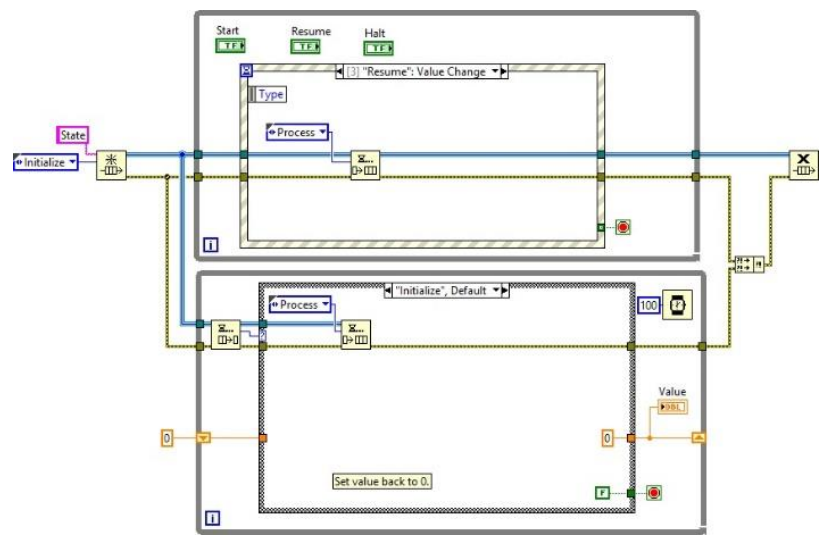

Figura 11 Estructura de programación Queue SM. Fuente: Elaboración Propia

\section{Interfaz de Usuario}

Se desarrolló una interfaz de usuario por medio de un menú, en donde se selecciona el corte a realizar: cortes en forma de cuadrado, circulares y mediante archivos CAD y cortes manuales, véase Figura 12.

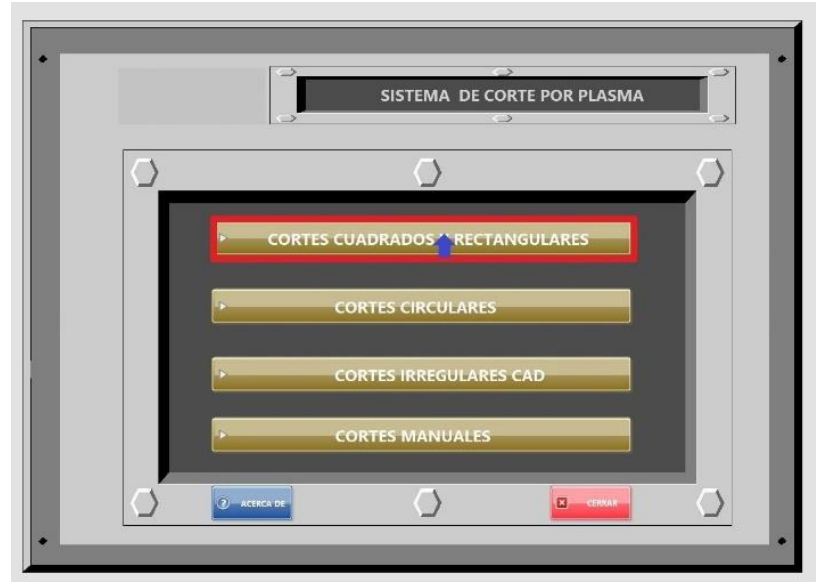

Figura 12 Interfaz principal de Usuario Fuente: Elaboración Propia 


\section{Selección de cortes a realizar}

Cada sección de corte se diseñó con todos los elementos necesarios para brindar al usuario una mayor comodidad y ofrecerle seguridad durante la operación.

Archivos CAD: Fue diseñada una sección para cualquier diseño o modelo previamente diseñado mediante algún software conocido.

Cortes manuales: Fue diseñada una sección para realizar cortes de manera manual, teniendo un mayor control de toda la máquina, considerando todos los controles necesarios.

Cortes circulares, cuadrados y rectangulares: Esta sección para realizar cortes en forma de cuadros y círculos, solo que cada una cuenta con pequeños cambios. Para el de forma circular pide un radio para ejecutar el corte, a diferencia en la sección para cortes cuadrados, el cual requiere de dos parámetros largo y ancho.

\section{Pruebas de funcionamiento}

Se realizaron pruebas para conocer el funcionamiento de la máquina y la interfaz diseñada de cada forma de corte.

Fue diseñada una figura mediante en un software CAD para ejecutarse mediante la interfaz y observar el modo de operación de los motores, así como verificar el funcionamiento de los paros de emergencia. En la Figura 13 se muestra el diseño realizado en AUTOCAD para realizar la prueba de funcionamiento.

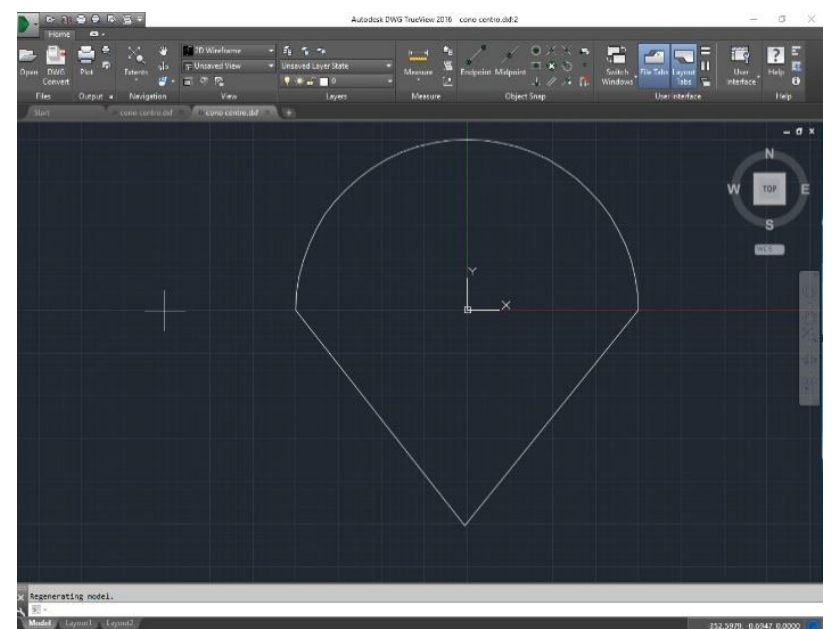

Figura 13 Diseño en AUTOCAD.

Fuente: Elaboración Propia
El diseño fue cargado en la interfaz desarrollada, como se muestra en la Figura 14 y se colocó un plumón para observar la trayectoria que generan los motores véase Figura 15.

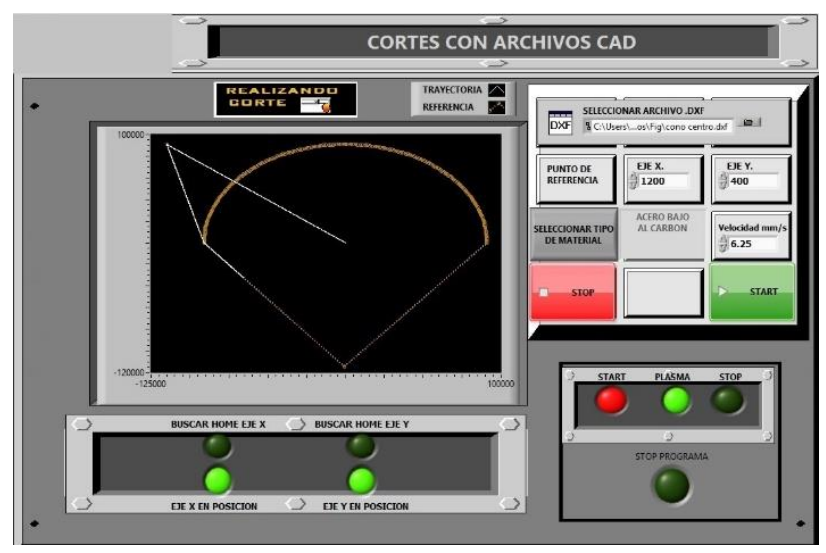

Figura 14 Diseño cargado en la interfaz. Fuente: Elaboración Propia

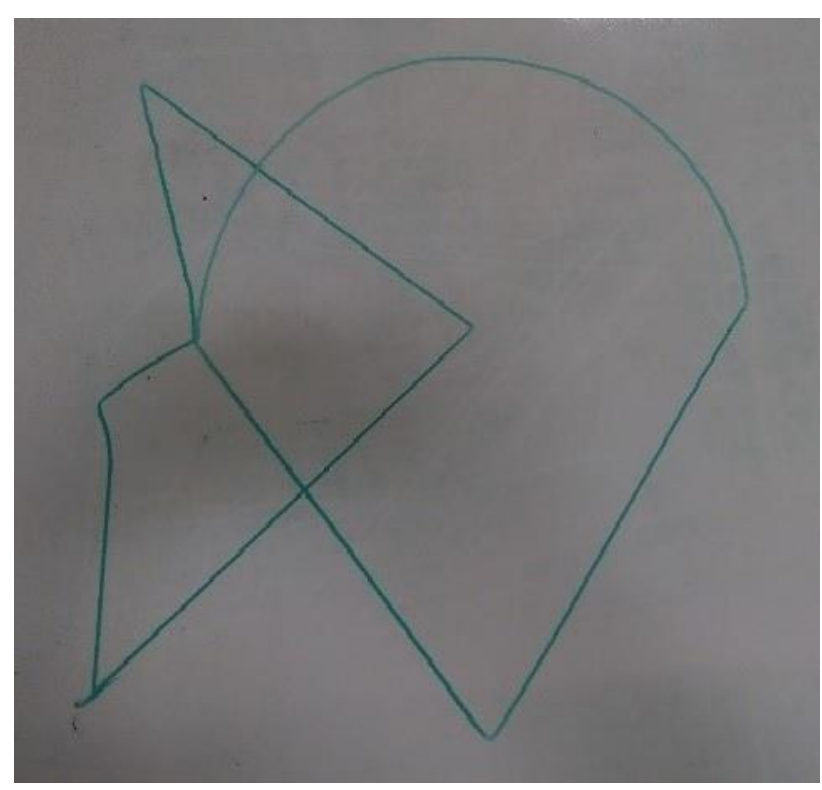

Figura 15 Trayectoria generada por los motores. Fuente: Elaboración Propia

\section{Paros de emergencia}

Se probaron los paros de emergencia para garantizar la seguridad ante cualquier accidente, en la Figura 16 se muestra el mensaje que aparece al presionarse el interruptor de emergencia. 


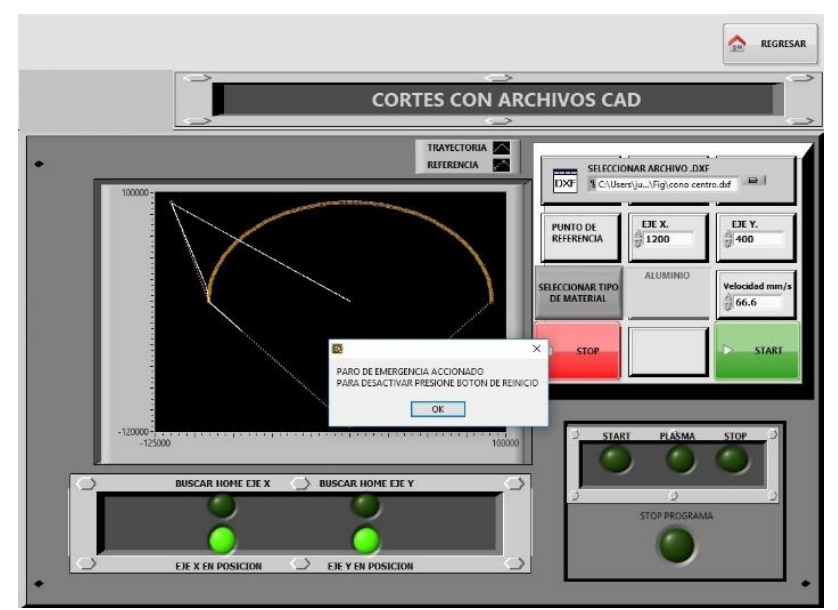

Figura 16 Paro de emergencia activado. Fuente: Elaboración Propia

De la misma manera se probaron los sensores de límite, los cuales como se observa en la Figura 17 se muestra un mensaje indicando lo sucedido.

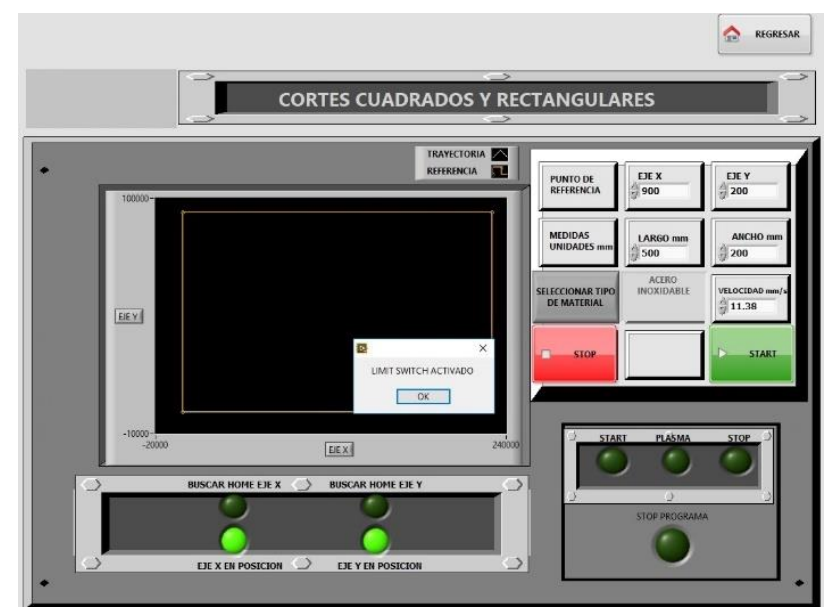

Figura 17 Sensor de límite activado. Fuente: Elaboración Propia

\section{Resultados}

Al realizar los cortes de modo manual, los resultados que se obtienen no son de una buena calidad, debido a que la precisión con la que se ejecutan los cortes depende del operador, lo que implica realizar un trabajo adicional para eliminar rebabas y mejorar estéticamente el corte.

La necesidad de realizar los cortes con una mejor calidad y rapidez fue necesario utilizar mecanismos, circuitos eléctricos y electrónicos para facilitar la tarea de los operadores para aumentar el tiempo de productividad, el cual se logró con el sistema desarrollado con las especificaciones requeridos como son: cortes de piezas con formas regulares e irregulares.
Entre las principales ventajas de la automatización de la mesa de corte con respecto al modo manual, se encuentran:

\section{A. Mayor precisión}

El acabado obtenido mediante este proceso es prácticamente definitivo, a diferencia del modo manual, que requiere mucho más trabajo adicional.

\section{B. Mayor calidad y rapidez}

Es posible conseguir una velocidad constante de corte. Por otro lado, no necesita trabajo adicional para eliminar imperfectos. Para constatar la efectividad del funcionamiento del sistema desarrollado se realizaron pruebas de corte en placas de acero. En la Figura 18 puede observarse como se ejecuta el corte.

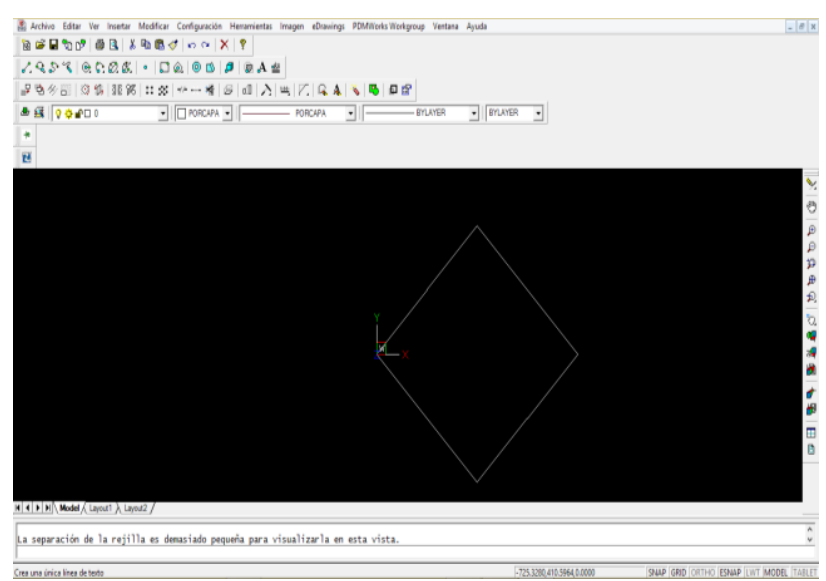

Figura 18 Sistema en funcionamiento.

Fuente: Elaboración Propia

En la Figura 19 se aprecia el corte de una pieza previamente diseñada y con buena precisión para su validación experimental.

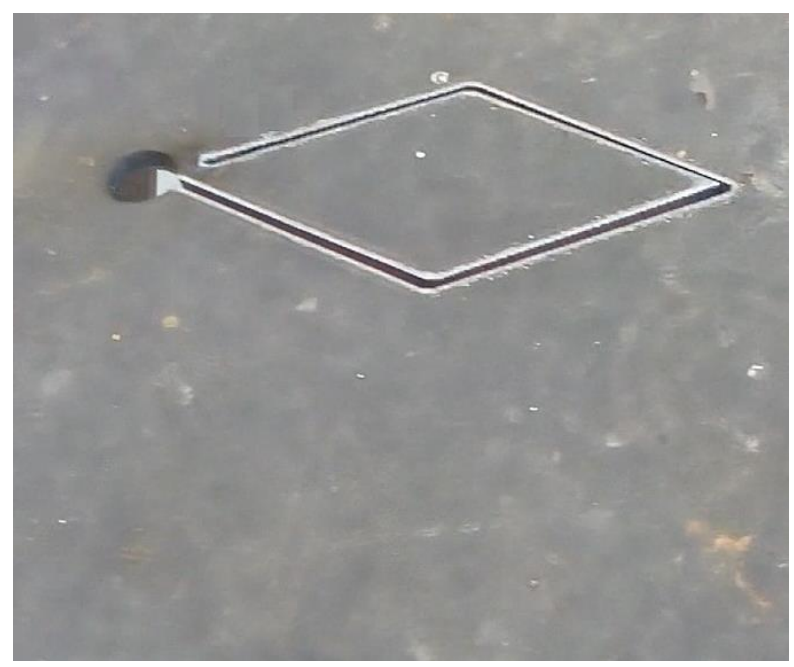

Figura 19 Corte de una pieza Fuente: Elaboración Propia

CASTILLO-QUIROZ, Gregorio, VARGAS-CRUZ, Juan Javier, REYES-LEON, Ivan y GONZAGA-LICONA, Elisa. Mesa de corte por plasma automatizada mediante LabVIEW. Revista de Aplicaciones de la Ingeniería. 2019. 
La contribución de este trabajo es de gran apoyo, debido a que se generó una programación que descifra algoritmos que generan los softwares de diseño. Dicha programación permite modificar y mejorar y posee una gran ventaja a comparación de softwares comerciales, aunque algunos de ellos son de uso libre pero no permiten modificarse.

\section{Agradecimiento}

Al Instituto Tecnológico Superior de Huauchinango y a la carrera de Ingeniería Mecatrónica por el apoyo y la confianza depositada para llevar a cabo este proyecto.

\section{Conclusiones}

La culminación e implementación del proyecto desarrollado es de gran ayuda, con la automatización de la mesa para corte por plasma tuvo un impacto favorable en la reducción de los tiempos de operación de trabajo, esto debido a que anteriormente los cortes se realizaban manualmente.

Este sistema se desempeña adecuadamente para las necesidades del usuario, lo cual cumple con las expectativas que se tenían previstas desde un inicio, garantizando un buen funcionamiento y medidas de seguridad durante su operación como lo sería con cualquier otro sistema de corte comercial. Es decir el sistema cumplió con los objetivos planteados.

\section{Referencias}

¿Qué es el corte por plasma CNC? (Julio de 2019) Obtenido de SIDECO: https://sideco.com.mx/que-es-el-corte-porplasma-cnc/

Articulo técnico corte y plasma (Julio de 2019). Obtenido de Praxair: https://microsites.praxair.es/media/PDF/corte\% 20plasma_es.pdf

Condori Cosi, M. C. (2019). Módulo de entrenamiento del simulador de soldadura en el aprendizaje significativo de los estudiantes del III ciclo de Mantenimiento de Maquinaria de Planta-TECSUP.

Corte por Plasma. (Julio de 2019). Obtenido de EcuRed

https://www.ecured.cu/Corte_por_plasma
Escuela Europea de Formación Empresarial, S. A. (2019). Preparación de herramientas, máquinas y equipos para la confección de productos textiles. TCPF0309. IC Editorial.

García Tsai, Montero Juan, Calle Wilson. Plasma: una tecnología de gran potencial para la industria y la ciencia. Revista de ciencia y tecnología Ingenius (págs. 66-72). Ecuador: Universidad Politécnica Salesiana. http://www.ni.com/labview/esa/

Importancia del CAD (Julio de 2019) Obtenido de EcuRed: https://www.ecured.cu/Dise\%C3\%B1o_asistido _por_computadora

Instruments, N. (Julio de 2019). Modulacion de Ancho de Pulso (PWM). Obtenido de http://www.ni.com/tutorial/2991/en/

Instruments, N. (Julio de 2019). National Instruments. Obtenido de La evolución del corte con plasma (Julio de 2019). Obtenido de https://www.thefabricator.com/spanish/laevoluci-n-del-corte-con-plasma

LabVIEW SoftMotion (Julio de 2019). National Instruments. Obtenido de http://www.ni.com/es$\mathrm{mx} / \mathrm{shop} / \mathrm{select} / \mathrm{labview}$-softmotion-module

Motor Drives (Julio de 2019). National Instruments. Obtenido de http://www.ni.com/pdf/manuals/372902b.pdf

NI 7340 Motion Controller (Julio de 2019). National Instruments. Obtenido de http://www.ni.com/pdf/manuals/370838b.pdf

NI MAX (noviembre de 2018). National Instruments. Obtenido de https://knowledge.ni.com/KnowledgeArticleDet ails?id=kA00Z000000P9KBSA0\&l=es-MX

Palanco-Lopez, S. (2019). Aplicaciones de láseres de potencia a la soldadura de aceros inoxidables.

$\mathrm{R}$

odríguez, T. J. (2019). Soldagem dissimilar autógena com laser pulsado Nd: YAG dos aços Rodríguez-Rabadán, Mateos-Aparicio, C. (2019). Desarrollo y aplicación industrial del software de seguridad de comprobación dual en un robot FANUC. 
Selección de gas plasma (Julio 2019). Obtenido de Hypertherm: https://www.hypertherm.com/es/learn/articles/il lustrated-guide-to-plasma-gasselection/?region=NART

superduplex UNS S32750 e superaustenítico AISI 904 corte por plasma automatizada mediante LabVIEW. Revista de Aplicaciones de la Ingeniería. 2019. 